\title{
THE IMPEDANCE OF THE CERAMIC CHAMBER IN J-PARC
}

\author{
Y. Shobuda, JAERI, Tokai_mura, Ibaraki, Japan \\ Y. H. Chin, K. Ohmi, T. Toyama, KEK ,Tsukuba, Ibaraki, Japan
}

\section{Abstract}

We calculate the impedance of the ceramic chamber inside of which is coated with TiN and outside of which is $\mathrm{Cu}$ shielded.

\section{INTRODUCTION}

Space charge impedance of rf-shielding wires with external ceramic and conducting pipes were studied by $\mathrm{T}$. F. Wang et. al.[1]. This system corresponds to the beam duct adopted in ISIS.

In J-PARC[2] the ceramic chamber is surrounded by $\mathrm{Cu}$-stripes due to technical reasons. The inner surface of the chamber is coated with TiN to suppress the secondary electron emission. Tsutsui and Lee studied this system by replacing $\mathrm{Cu}$-stripes to the conducting beam pipe[3,4]. In this paper we deal with $\mathrm{Cu}$-stripes as rf-shield wires, and compare the results with the previous results. The resistivity of these rf-shield wires and the TiN coating is considered.

\section{THE LONGITUDINAL IMPEDANCE}

In this section we calculate the longitudinal impedance of the ceramic chamber. We use the cylindrical coordinate $(r, \theta, z)$. A source particle $q$ moves along the $\mathrm{z}$ direction with velocity $\beta c$ at an offset of $r=r_{b}, \theta=\theta_{\beta}$. The charge density $\rho$ is expressed as,

$$
\begin{aligned}
& \rho=\frac{q}{r_{b}} \delta\left(r-r_{b}\right) \delta_{p}\left(\theta-\theta_{b}\right) \delta(z-\beta c t) \\
& =\sum_{m=0}^{\infty} \int \frac{d k}{2 \pi} \frac{i_{m} \cos m\left(\theta-\theta_{b}\right)}{\pi r_{b}^{m+1}\left(1+\delta_{m 0}\right)} \delta\left(r-r_{b}\right) e^{i k(z-\beta c t)}, \\
& i_{m}=q r_{b}^{m} .
\end{aligned}
$$

Here we introduce multipole charge density $\rho_{\mathrm{m}}$ :

$$
\rho_{m}=\frac{i_{m} \cos m\left(\theta-\theta_{b}\right)}{\pi r_{b}^{m+1}\left(1+\delta_{m 0}\right)} \delta\left(r-r_{b}\right) e^{i k(z-\beta c t)}
$$

The field driven by the charge density $\rho$ is obtained by the superposition of the field come from this multipole charge density.

The system we consider is described in Fig.1.The field is calculated by solving the Helmholz equations for the scalar potential except the TiN coating region. When we deal with the TiN coating region, we solve the equation for the vector potential. The solutions of this system where $\mathrm{e}^{\mathrm{ik}(\mathrm{z}-\mathrm{\beta ct})}$ is omitted are written as,

$$
\begin{aligned}
& E_{z}=-\frac{i k b_{m}}{\gamma^{2}}\left(\cos \left(\theta-\theta_{b}\right) \frac{K_{m}\left(\bar{k} r_{b}\right)}{I_{m}\left(\bar{k} r_{b}\right)} I_{m}(\bar{k} r)\right. \\
& \left.+\sum_{p=-\infty}^{\infty} K_{m-p N(k} \bar{k} a F_{m-p N}(k) \cos \left[(m-p N) \theta-m \theta_{b}\right] I_{m-p N}\left(\bar{k} r_{b}\right)\right) \text { for } \quad r<r_{b},
\end{aligned}
$$

$$
\begin{aligned}
& E_{z}=-\frac{i k b_{m}}{\gamma^{2}}\left(\cos \left(\theta-\theta_{b}\right) K_{m}(\bar{k} r)\right. \\
& \left.+\sum_{p=-\infty}^{\infty} K_{m-p N}\left(\bar{k} a_{I}\right) F_{m-p N}(k) \cos \left[(m-p N) \theta-m \theta_{b}\right] I_{m-p N}(\bar{k} r)\right) \\
& \text { for } \quad r_{b}<r<a_{I},
\end{aligned}
$$

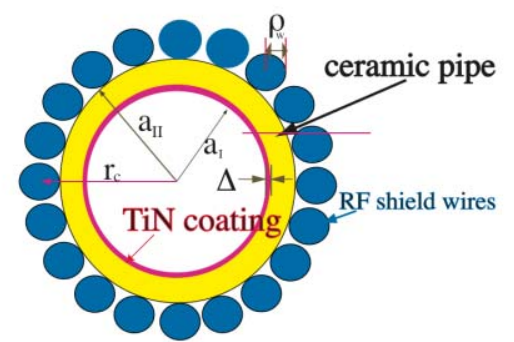

Fig.1: The ceramic chamber outside of which is surrounded by rf-shield wires. The inner surface is coated with TiN.

$$
\begin{aligned}
& E_{z}=i k b_{m} \beta c \sum_{p=-\infty}^{\infty}\left(K_{m-p N}\left(\kappa_{T i N}\left(a_{I}+\Delta\right)\right) A_{m-p N} I_{m-p N}\left(\kappa_{T i N} r\right)\right. \\
& \left.+I_{m-p N}\left(\kappa_{T i N} a_{I}\right) B_{m-p N} K_{m-p N}\left(\kappa_{T i N} r\right)\right) \cos \left[(m-p N) \theta-m \theta_{b}\right] \\
& \text { for } \quad a_{I}<r_{b}<a_{I}+\Delta,
\end{aligned}
$$

$E_{z}=-\frac{i k b_{m}}{\gamma_{1}^{2}}\left(\frac{1}{\varepsilon_{1}} \sum_{p=-\infty}^{\infty} I_{m-p N}\left(\bar{k}_{1} a_{I}\right) F_{m-p N}^{(1)}(k) \cos \left[(m-p N) \theta-m \theta_{b}\right] K_{m-p N}\left(\bar{k}_{1} r\right)\right.$

$\left.+\frac{1}{\varepsilon_{1}} \sum_{p=-\infty}^{\infty} K_{m-p N}\left(\bar{k}_{1} a_{I I}\right) F_{m-p N}^{(2)}(k) \cos \left[(m-p N) \theta-m \theta_{b}\right] I_{m-p N}\left(\bar{k}_{1} r\right)\right)$

for $a_{I}+\Delta<r_{b}<a_{I I}$,

$E_{z}=-\frac{i k b_{m}}{\gamma^{2}}\left(\sum_{p=-\infty}^{\infty} I_{m-p N}\left(\bar{k}_{1} a_{I I}\right) F_{m-p N}^{(3)}(k) \cos \left[(m-p N) \theta-m \theta_{b}\right] K_{m-p N}(\bar{k} r)\right.$

$\left.-\frac{1}{2} \sum_{n=-\infty}^{\infty} \frac{I_{n}\left(\bar{k} \rho_{W}\right)}{K_{n}\left(\bar{k} \rho_{W}\right)} N K_{m+n}\left(\bar{k} r_{c}\right)\left(e^{i m\left(\bar{m} \Delta-\theta_{b}\right)} Q_{n}(r) P_{n}+e^{-i m\left(\bar{m} \Delta-\theta_{b}\right)} Q_{n}^{*}(r) P_{n}\right)\right)$

for $a_{I I}<r$,

where

$Q_{n}=\sum_{p=-\infty}^{\infty} K_{n+m-p N}\left(\bar{k} r_{c}\right) I_{m-p N}(\bar{k} r) e^{i(m-p N)(\theta-\bar{m} \Delta)}$,

for $r<r_{c}, r$ and $r_{c}$ are exchanged for $r>r_{c}, r_{c}$ is the position of rf-wire, $\varepsilon_{1}$ is the relative dielectric constant of ceramic, $\mu_{1}$ is the relative permeability of ceramic, the wave number $\mathrm{k}$ and the frequency $\mathrm{f}$ is related by $\mathrm{k}=2 \pi \mathrm{f} / \beta \mathrm{c}, \bar{k}=k / \gamma$, $\gamma_{1}=1 / \sqrt{1-\beta^{2} \varepsilon_{1} \mu_{1}}, \sigma_{\mathrm{TiN}}$ is the conductivity of $\mathrm{TiN}, \sigma_{\mathrm{RF}}$ is the conductivity of rf-wire, $b_{m}=i_{m} I_{m}\left(\bar{k}_{b}\right) / \varepsilon_{0} \pi r_{b}^{m}\left(1+\delta_{m 0}\right), \delta_{\mathrm{m}, 0}$ is Kroneker $\delta, \Delta$ is the width of TiN coating, $a_{I}+\Delta$ is the inner radius of the ceramic, $a_{\text {II }}$ is the outer radius of the 
ceramic, $\varepsilon_{\mathrm{RF}}$ is the relative dielectric constant of rf-wire which is given by $1+i \sigma_{\mathrm{RF}} \mathrm{Z}_{0} / \mathrm{k} \beta[5,6], \varepsilon_{\mathrm{TiN}}$ is the relative dielectric constant of TiN which is given by $1+\mathrm{i} \sigma_{\mathrm{TiN}} \mathrm{Z}_{0} / \mathrm{k} \beta[5,6], \kappa_{R F}=\sqrt{k^{2}-\varepsilon_{R F} \mu_{R F} k^{2} \beta^{2}}, \kappa_{T i N}=\sqrt{k^{2}-\varepsilon_{T i N} \mu_{T i N} k^{2} \beta^{2}}$,

and $\mathrm{F}_{\mathrm{m}-\mathrm{pN}}, \mathrm{F}_{\mathrm{m}-\mathrm{pN}}{ }^{(1)}, \mathrm{F}_{\mathrm{m}-\mathrm{pN}}{ }^{(2)}, \mathrm{F}_{\mathrm{m}-\mathrm{pN}}{ }^{(3)}, \mathrm{P}_{\mathrm{n}}, \mathrm{A}_{\mathrm{m}-\mathrm{pN}}$ and $\mathrm{B}_{\mathrm{m}-\mathrm{pN}}$ are arbitrary coefficients.

Let us consider the field inside of the rf-shield wire. Following T. F. Wang, we use local coordinate $\left(\rho, \phi_{\bar{m}}, z\right)$, where $\bar{m}$ denotes the index of the ordering of rf-wire. Since the field inside of the rf-wire satisfies

$$
\left(\frac{\varepsilon_{R F} \mu_{R F}}{c^{2}} \frac{\partial^{2}}{\partial t^{2}}-\Delta\right) \vec{A}=0,
$$

where $\Delta=\nabla \cdot(\nabla \cdot)-\nabla \times(\nabla \times), \mathrm{A}_{\mathrm{z}}$ can be written as,

$A_{z}^{R F}=\frac{b_{m}}{2} \sum_{l}\left(c_{l} e^{i m\left(\bar{m} \Delta-\theta_{b}\right)}+c_{-l} e^{-i m\left(\bar{m} \Delta-\theta_{b}\right)}\right) I_{l}\left(\kappa_{R F} \rho_{\bar{m}}\right) e^{i l \phi_{\bar{m}}}$

where $c_{1}$ are the arbitrary coefficients. According to matching conditions, $\mathrm{P}_{\mathrm{n}}, \mathrm{c}_{\mathrm{l}}$ and $\mathrm{F}_{\mathrm{m}}{ }^{(3)}$ are related as follows, $\sum_{n=-\infty}^{\infty} \frac{I_{n}\left(\bar{k} \rho_{W}\right)}{K_{n}\left(\bar{k} \rho_{W}\right)} N K_{m+n}\left(\bar{k} r_{c}\right) P_{n} d_{n, l}$

$=\sum_{p=-\infty}^{\infty} I_{m-p N}\left(\bar{k} a_{I I}\right) F_{m-p N}^{(3)} K_{m+l-p N}\left(\bar{k} r_{c}\right) I_{l}\left(\bar{k} \rho_{W}\right)+\gamma^{2} \beta c I_{l}\left(\kappa_{R F} \rho_{W}\right) c_{l}$,

$\frac{\beta}{c}\left(1-\frac{1}{\varepsilon_{R F} \mu_{R F} \beta^{2}}\right) \sum_{p=-\infty}^{\infty} I_{m-p N}\left(\bar{k} a_{I I}\right) F_{m-p N}^{(3)} K_{m+l-p N}\left(\bar{k} r_{c}\right) \frac{1}{2}\left(I_{l+1}\left(\bar{k} \rho_{W}\right)+I_{l-1}\left(\bar{k} \rho_{W}\right)\right)$

$-\frac{\beta}{c}\left(1-\frac{1}{\varepsilon_{R F} \mu_{R F} \beta^{2}}\right) \sum_{n=-\infty}^{\infty} \frac{I_{n}\left(\bar{k} \rho_{W}\right)}{K_{n}\left(\bar{k} \rho_{W}\right)} N K_{m+n}\left(\bar{k} r_{c}\right) P_{n} d_{n, l}^{\prime}\left(\rho_{W}\right)$

$=\frac{1}{\mu_{R F}} c_{l} \frac{\kappa_{R F}}{2}\left(I_{l+1}\left(\kappa_{R F} \rho_{W}\right)+I_{l-1}\left(\kappa_{R F} \rho_{W}\right)\right)$,

where $\rho_{\mathrm{W}}$ is the radius of rf-wire, $\mathrm{d}_{\mathrm{n}, \mathrm{l}}$ is the Fourier coefficients of $\mathrm{Q}_{\mathrm{n}}$ :

$$
Q_{n}=\sum_{l=-\infty}^{\infty} d_{n, l}\left(\rho_{\bar{m}}\right) e^{i l \phi_{\bar{m}}},
$$

prime at $d_{n, 1}$ in Eq.(12) means taking the derivative of $\rho_{\bar{m}}$.

All arbitrary coefficients: $\mathrm{F}_{\mathrm{m}-\mathrm{pN}}, \mathrm{F}_{\mathrm{m}-\mathrm{pN}}{ }^{(1)}, \mathrm{F}_{\mathrm{m}-\mathrm{pN}}{ }^{(2)}, \mathrm{F}_{\mathrm{m}-\mathrm{pN}}{ }^{(3)}$, $\mathrm{P}_{\mathrm{n}}, \mathrm{c}_{\mathrm{l}}, \mathrm{A}_{\mathrm{m}-\mathrm{pN}}$ and $\mathrm{B}_{\mathrm{m}-\mathrm{pN}}$ are decided by Eqs.(11),(12) and the matching conditions at $r=a_{I}, r=a_{I}+\Delta, r=a_{I I}: E_{z}$, $\mathrm{H}_{\theta}$ and the normal component of $\mathrm{D}$ are continuous. In our calculation, we only consider the lowest order.

Since we find arbitrary coefficients, we can calculate the longitudinal impedance. According to Eqs.(3) and (4), $E_{z}$ for the cylindrical beam whose radius is $\sigma$ is expressed as,

$$
\begin{aligned}
& E_{z}=-\frac{c Z_{0}}{\pi \sigma^{2}} \frac{i k}{\gamma^{2}}\left(\int_{r}^{\sigma} d r_{b} r_{b} K_{0}\left(\bar{k} r_{b}\right) I_{0}(\bar{k} r)+\int_{0}^{r} d r_{b} r_{b} I_{0}\left(\bar{k} r_{b}\right) K_{0}(\bar{k} r)\right) \\
& -\frac{c Z_{0}}{\pi \sigma^{2}} \frac{i k}{\gamma^{2}} \int_{0}^{\sigma} d r_{b} I_{0}\left(\bar{k} r_{b}\right) \sum_{p=-\infty}^{\infty} K_{p N}\left(\bar{k} a_{I}\right) F_{p N}(k) \cos [p N \theta] I_{p N}(\bar{k} r) \\
& =-\frac{c Z_{0}}{\pi \sigma^{2}} \frac{i k}{\gamma^{2}}\left(\frac{1}{\bar{k}^{2}}-\frac{\sigma I_{0}(\bar{k} r) K_{1}(\bar{k} \sigma)}{\bar{k}}\right)-\frac{c Z_{0}}{\pi \sigma^{2}} \frac{i k}{\gamma^{2}} \frac{\sigma I_{1}(\bar{k} \sigma)}{\bar{k}} \sum_{p=-\infty}^{\infty} K_{p N}\left(\bar{k} a_{I}\right) F_{p N}(k) \cos [p N \theta] I_{p N}(\bar{k} r) .
\end{aligned}
$$

By taking an average for $\mathrm{r}$, we obtain

$$
\frac{Z_{\|}}{n}=2 \frac{Z_{0}}{\sigma^{2}} \frac{i}{\gamma \sqrt{\gamma^{2}-1}} \frac{1}{\bar{k}^{2}}\left(1-2 K_{1}[\bar{k} \sigma] I_{1}[\bar{k} \sigma]+2 I_{1}^{2}[\bar{k} \sigma] K_{0}\left[\bar{k} a_{I}\right] F_{0}(k)\right) \text {. }
$$

Space charge impedance is automatically included in Eq.(15). In order to define the impedance of the ceramic chamber, we have to subtract the space charge part. By considering the system that the perfect conducting pipe exists at $\mathrm{r}=\mathrm{a}_{\mathrm{I}}$, the space charge impedance is calculated as follows,

$$
\frac{Z_{\|}}{n}=-2 \frac{Z_{0}}{\sigma^{2}} \frac{i}{\beta k^{2}}\left(1-2 K_{1}[\bar{k} \sigma] I_{1}[\bar{k} \sigma]-2 \frac{K_{0}\left[\bar{k} a_{I}\right]}{I_{0}\left[\bar{k} a_{I}\right]} I_{1}^{2}[\bar{k} \sigma]\right) \text {. }
$$

Here we should notice that Eq.(16) reproduces

$\rightarrow-\frac{i Z_{0}}{2 \beta \gamma^{2}}\left(\frac{1}{2}-2 \log \left[\frac{\sigma}{a_{I}}\right]\right)$

for large $\gamma[7]$. From now on, we assume that space charge part included in Eq.(15) is given by Eq.(16). We define the impedance for the ceramic chamber by subtracting Eq.(16) from Eq.(15).

Previously, Lee obtained the analytic formula of the longitudinal impedance per a unit length for the system described in Fig.2[4]. This can be rewritten as follows,

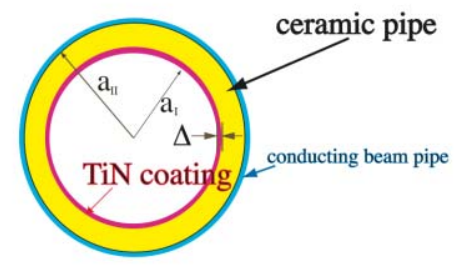

Fig.2: The ceramic chamber outside of which is surrounded by the conducting beam pipe.

$$
\frac{Z}{n}=\frac{Z_{0}}{k \beta a_{I}\left(-\frac{\varepsilon_{1}}{\left(\varepsilon_{1} \beta^{2}-1\right) k a_{I} \ln a_{I I} / a_{I}} i-Z_{0} \Delta \sigma_{T i N}\right)},
$$

where small $\mathrm{k}$ approximation is applied. In Fig. 3 we present the results calculated by Eq.(17), and the rigorous numerical results where small $\mathrm{k}$ approximation is not applied. We find that these two results are good agreement as $\gamma$ becomes larger.
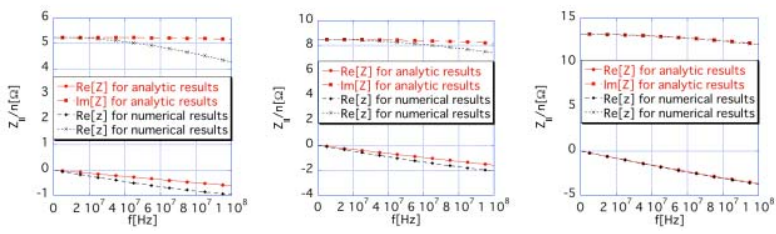

Fig. 3: The longitudinal impedance of the ceramic chamber for Fig. 2 is shown. The analytic results and rigorous numerical results are compared. The left figure corresponds to $\gamma=1.181$, the middle figure $\gamma=1.4$, the right figure $\gamma=4 . \quad r_{c}=0.1945[\mathrm{~m}], \quad \varepsilon_{1}=10, \quad \mu_{1}=1, \quad a_{2}=0.189[\mathrm{~m}]$, $\mathrm{a}_{1}=0.1815[\mathrm{~m}], \sigma_{\mathrm{TiN}}=6 \times 10^{6}[\Omega \cdot m], \sigma_{\mathrm{RF}}=5 \times 10^{7}[\Omega \cdot m]$, the beam size $\sigma=\sqrt{216 \pi \times 10^{-6} \times 10}, \quad \Delta=10[\mathrm{~nm}], \mathrm{N}_{\mathrm{W}}=110$. $\operatorname{Re}\left[\mathrm{Z}_{\|}\right]<0$ means deceleration.

Let us compare our results of the longitudinal impedance with the previous results. In Fig.4, we compare the longitudinal impedances for Fig.1 with 
numerical results for Fig.2. These two results are good agreement as $\gamma$ becomes larger.
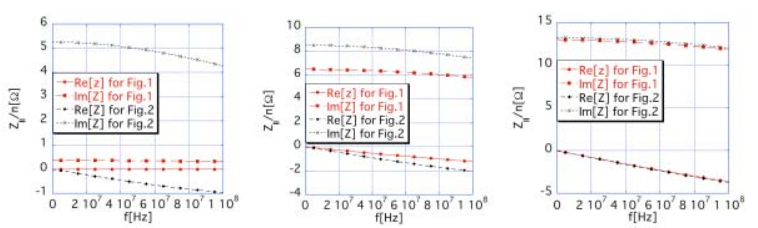

Fig.4: The longitudinal impedance of the ceramic chamber is shown. The results for Fig.1 and the rigorous numerical results for Fig.2 are compared. The left figure corresponds to $\gamma=1.181$, the middle figure $\gamma=1.4$, the right figure. The parameters: $r_{c}, \sigma_{\mathrm{TiN}}, \varepsilon_{1}, \mu_{1}, \mathrm{a}_{1}, \mathrm{a}_{2}, \Delta$ and $\sigma_{\mathrm{TiN}}$ are the same as those described in Fig.3. Other parameters are given by $\sigma_{\mathrm{RF}}=5 \times 10^{7}[\Omega \cdot m], \rho_{\mathrm{W}}=2.75[\mathrm{~mm}], \mathrm{N}_{\mathrm{W}}=110$. $\operatorname{Re}\left[Z_{\|}\right]<0$ means deceleration.

\section{THE TRANSVERSE IMPEDANCE}

The transverse force $F_{x, y}$ is calculated by PanofskyWenzel theorem using $\mathrm{E}_{z}$, while the transverse force can be written by the transverse impedance $Z_{1}$ as follows,

$$
F_{x, y}=i c \beta i_{1}\left(\frac{d r}{d r} \cos \left(\theta-\theta_{b}\right)-r \frac{d \theta}{d \vec{r}} \sin \left(\theta-\theta_{b}\right)\right) \frac{Z_{1}}{2 \pi R},
$$

where $2 \pi R$ is the circumference of our ring [8]. Therefore, we obtain

$$
Z_{1, \text { with } S P}=i \frac{I_{1}\left[\bar{k} r_{b}\right] Z_{0} k R}{\beta \gamma^{3} r_{b}}\left(\frac{K_{1}\left[\bar{k} r_{b}\right]}{I_{1}\left[\bar{k} r_{b}\right]}+K_{1}\left[\bar{k} a_{1}\right] F_{1}\right) .
$$

As in the previous section, the space charge impedance is also included in this impedance. Since the transverse space charge impedance for the system that the perfect conducting pipe exists at $\mathrm{r}=\mathrm{a}_{\mathrm{I}}$, can be written as,

$Z_{1, S P}=i \frac{I_{1}\left[\bar{k} r_{b}\right] Z_{0} k R}{\beta \gamma^{3} r_{b}}\left(\frac{K_{1}\left[\bar{k} r_{b}\right]}{I_{1}\left[\bar{k} r_{b}\right]}-\frac{K_{1}\left[\bar{k} a_{1}\right]}{I_{1}\left[\bar{k} a_{1}\right]}\right)$,

we define the transverse impedance for the ceramic chamber as follows,

$$
Z_{1}=i \frac{I_{1}\left[\bar{k} r_{b}\right] Z_{0} k R}{\beta \gamma^{3} r_{b}}\left(\frac{K_{1}\left[\bar{k} a_{1}\right]}{I_{1}\left[\bar{k} a_{1}\right]}+K_{1}\left[\bar{k} a_{1}\right] F_{1}\right),
$$

by subtracting Eq.(20) from Eq.(19). Here we should notice that Eq.(20) reproduces

$\rightarrow \rightarrow i Z_{0} \frac{R}{\beta \gamma^{2}}\left(\frac{1}{r_{b}^{2}}-\frac{1}{a_{1}^{2}}\right)$,

for large $\gamma[8]$.

Let us compare the transverse impedances of the ceramic chamber for Fig.1 with those for Fig. 2 as in the longitudinal case. Results are represented in Fig.5. Two results are good agreement as $\gamma$ becomes larger.
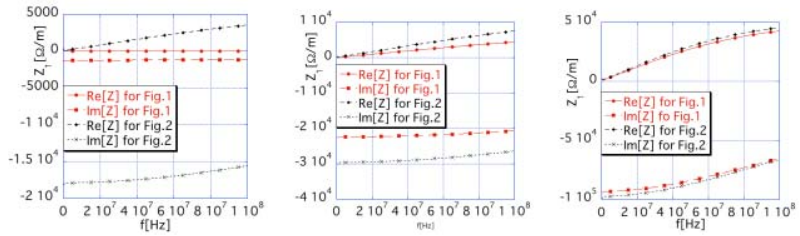

Fig.5: The transverse impedance of the ceramic chamber is shown. The results for Fig.1 and those for Fig.2 are compared. The left figure corresponds to $\gamma=1.181$, the middle figure $\gamma=1.4$, the right figure $\gamma=4$. The parameters : $\mathrm{r}_{\mathrm{c}}, \sigma_{\mathrm{TiN}}, \varepsilon_{1}, \mu_{1}, \mathrm{a}_{1}, \mathrm{a}_{2}, \Delta, \sigma_{\mathrm{TiN}}, \sigma_{\mathrm{RF}}, \rho_{\mathrm{W}}$ and $\mathrm{N}_{\mathrm{W}}$ are the same as those described in Fig.4.

\section{CONCLUSIONS}

The longitudinal and transverse impedance of the ceramic chamber inside of which is coated by TiN and outside of which is $\mathrm{Cu}$-shielded are calculated. Previously, the impedance for this system was calculated by simplifying the system: replacing the $\mathrm{Cu}$-stripes to the perfect conducting pipe. The results obtained here are compared with those previously obtained results. As $\gamma$ becomes larger, those two results coincide each other; this means that the approximation that replacing the rf-shield wires to the conducting beam pipe is quite appropriate for large $\gamma$.

\section{ACKNOWLEDGEMENTS}

The authors thank K. Yokoya, K. Takata, K. Oide and S. Machida for fruitful discussions. They also thank J-PARC members in JAERI/KEK.

\section{REFERENCES}

[1] T. F. Wang, S. S. Kurennoy and R. L. Gluckstern Physical Review S. T. 2001, p.104201

[2] Y.Yamazaki, Accelerator Technical Design Report for J-PARC, KEK Report 2002-13,JAERI-Tech 20003044

[3] H. Tsutsui, Internal Report (2001)

[4] S. Lee, RCS Resistive Wall Impedance, Internal Report (2003)

[5] N. W. Ashcroft and N. D. Mermin, Solid State Physics, Thomson learning (1976)

[6] J. D. Jackson, Classical Electrodynamics, John Wiley \&Sons Inc (1998)

[7] B. W. Zotter and S. A. Kheifets, Impedances and wakes in high-energy accelerators, Singapore, World Scientific, (1999)

[8] A. W. Chao, Physics of collective beam instabilities in high energy accelerators, New York, Wiley, (1993) 